\title{
RBIDS- IDS with Biometric Authentication and Trace Back Scheme for Enhanced Security
}

\author{
S.Gilbert Nancy \\ Lecturer \\ Department of IT \\ Thiagarajar College of Engg \\ Madurai, India
}

\author{
N. Nischala \\ Under Graduate \\ Department of IT \\ Thiagarajar College of Engg \\ Madurai, India
}

\begin{abstract}
There has been an increasing concern over the volume of attacks from international sources towards government and military networks. The next generation wars would all involve computers and network tools, rather than fighter planes and nuclear bombs. Our proposal is a range based intrusion detection system with a trace back scheme to survive against these next generation odds. The proposed system would use a biometric authentication scheme for data transmission internally within the network and the usual Intrusion Detection Scheme for data transmission with outside networks like internet. This biometric authentication also alleviates the problem that an outsider cannot enter the network by pretending to be an insider with spoofed IP address. In case an attack occurs, we use an Autonomous System level IP trace back to trace back the attack to its source. The advantages of our proposed system are:

1. The possibility of an attack from inside and outside the system is meager because the system has facilities to authenticate transmissions within the network and to detect malicious data from outside the network.

2. In case an attack occurs, the trace back scheme would trace back the attack to the source, thus enabling 'punish the guilt'.
\end{abstract}

The full text of the article is not available in the cache. Kindly refer the IJCA digital library at www.ijcaonline.org for the complete article. In case, you face problems while downloading the full-text, please send a mail to editor at editor@ijcaonline.org 